\title{
Potential Treatments for COVID-19 Related Cytokine Storm - Beyond Corticosteroids
}

\author{
Yi Miao ${ }^{1,2}$, Lei Fan ${ }^{1,2 *}$ and Jian-Yong $L^{1,2}$ \\ ${ }^{1}$ Department of Hematology, The First Affiliated Hospital of Nanjing Medical University, Jiangsu Province Hospital, Nanjing, \\ China, ${ }^{2}$ Key Laboratory of Hematology of Nanjing Medical University, Nanjing, China
}

Keywords: COVID-19, hemophagocytic lymphohistiocytosis, IL-6, ruxolitinib, tocilizumab

A novel virus, SARS-CoV-2, emerged in Wuhan in December 2019 and rapidly spread to other cities in China and other countries. Several studies have summarized the clinical characteristics and laboratory findings of patients with Corona Virus Disease 2019 (COVID-19) $(1,2)$. According to these studies, in addition to lung injury, damages involving other organs, which include liver, kidney, heart, and hemopoietic system, were also observed in some patients, suggesting the presence of systemic inflammation, and from the work by Huang et al. (1), we noted that elevation of various proinflammatory cytokines was present in patients infected with SARS-CoV2 , suggesting the possible existence of cytokine storm in a proportion of patients. Further, patients that require intensive care unit (ICU) admission showed higher concentrations of certain cytokines compared with those not requiring ICU admission, indicating that the levels of proinflammatory

OPEN ACCESS

Edited by:

Ji Ming Wang,

National Cancer Institute at Frederick,

United States

Reviewed by:

Paola Italiani,

National Research Council (CNR), Italy

*Correspondence:

Lei Fan

fanlei3014@126.com

Specialty section:

This article was submitted to

Cytokines and Soluble Mediators in

Immunity,

a section of the journal

Frontiers in Immunology

Received: 21 April 2020

Accepted: 03 June 2020

Published: 16 June 2020

Citation:

Miao Y, Fan L and Li J-Y (2020) Potential Treatments for COVID-19 Related Cytokine Storm - Beyond

Corticosteroids.

Front. Immunol. 11:1445.

doi: 10.3389/fimmu.2020.01445 cytokines were associated with disease severity. Further studies confirmed that levels of cytokines including interleukin (IL)-6 and IL-8 correlated with the disease severity of COVID-19 $(3,4)$. This phenomenon is not restricted to COVID-19, in the previous studies regarding the Middle East respiratory syndrome (MERS) and severe acute respiratory syndrome (SARS), higher levels of certain cytokines were associated with increased mortality (5, 6). For instance, high IL-6 concentration predicted mortality in patients with MERS (5). In patients infected with pathogenic human coronaviruses, cytokine storm contributes to acute lung injury and acute respiratory distress syndrome (ARDS) (7). Therefore, controlling the cytokine storm might be a strategy for treating patients with COVID-19, especially for those severe cases.

\section{POTENTIAL TREATMENTS}

Corticosteroids could be used to suppress the cytokine storm and have been used in some patients (1). However, based on the evidence from patients with MERS and ARDS, the use of corticosteroids did not provide a survival benefit but rather delayed the clearance of the virus, therefore, the systemic use of corticosteroids is not recommended by the $\mathrm{WHO}$ guidance (1). As a result, alternatives for dampening the overwhelming cytokine release are required.

As we know, the cytokine storm also occurs in other settings. In patients with leukemia or lymphoma who receive chimeric antigen receptor (CAR) T cells therapy, cytokine release syndrome (CRS) occurs during and after the infusion of CAR T cells (8). In patients receiving CAR T cells therapy, those with CRS had elevated concentrations of interferon $\gamma$, tumor necrosis factor $\alpha$, interleukin (IL)-1B, IL-2, IL-6, IL-7, IL-8, IL-10, IL-12, granulocyte macrophage colony stimulating factor (GM-CSF), and macrophage inflammatory protein (MIP)-1. The cytokine profile in CRS related to CAR T cells infusion is similar to that in cases of SARS-CoV-2 infection. The anti-IL-6 receptor antibody tocilizumab is effective in controlling CAR T cells infusion related CRS (response rate: $53-69 \%$ ) (9). The above evidence provides us with a rationale for using tocilizumab to manage the cytokine storm in patients with SARS-CoV-2 infection. Another rationale for using tocilizumab 
to treat COVID-19 is that IL- 6 does not enhance the antiviral immunity but decreases the antiviral immunity in patients with COVID-19. Diao et al. found that serum IL-6 was negatively correlated with T cell numbers (10). Mazzoni et al. found that the elevation of IL-6 serum levels was associated with the impairment of cytotoxic activity in patients with COVID-19, and the use of tocilizumab restored the cytotoxic potential of NK cells (11). Some studies involving off-label use of tocilizumab have shown the potential efficacy of this drug in the treatment of COVID19 (12-15).

Another potential drug that could be considered to treat cytokine storm is etoposide, which is used to deplete monocytes and suppress cytokine release in hemophagocytic lymphohistiocytosis (HLH) (16). It needs to be mentioned that, in SARS-CoV-infected mice, inflammatory monocytemacrophage responses were involved in causing lethal pneumonia, suggesting the importance of suppressing monocytemacrophage system in treating severe pneumonia related to SARS-CoV (17). The hyperactivation of monocytes/macrophages has been described in patients with COVID-19. Single-cell analysis of bronchoalveolar fluid revealed significantly increased proportions of mononuclear phagocytes in patients with COVID-19, especially those with severe disease. In patients with severe disease, these mononuclear phagocytes showed a predominance of inflammatory monocyte-derived macrophages (18). These macrophages could not only contribute to acute inflammation but also promote fibrosis generation. Additionally, a significant increase of $\mathrm{CD} 14^{+} \mathrm{CD} 16^{+}$monocytes was also detected in patients with severe COVID-19 (19). These $\mathrm{CD}_{14}{ }^{+} \mathrm{CD} 16^{+}$monocytes expressed IL- 6 and caused the acceleration of the inflammation. Therefore, etoposide could be used to inhibit the hyperactivation of monocytes/macrophages to suppress the overwhelming inflammation and ameliorate the pulmonary fibrosis. Other potential drugs for treating cytokine storm include the JAK1/2 inhibitor ruxolitinib, which is effective in inhibiting monocyte activation and cytokine

\section{REFERENCES}

1. Huang C, Wang Y, Li X, Ren L, Zhao J, Hu Y, et al. Clinical features of patients infected with 2019 novel coronavirus in Wuhan, China. Lancet. (2020) 395:497-506. doi: 10.1016/S0140-67362030183-5

2. Chen N, Zhou M, Dong X, Qu J, Gong F, Han Y, et al. Epidemiological and clinical characteristics of 99 cases of 2019 novel coronavirus pneumonia in Wuhan, China: a descriptive study. Lancet. (2020) 395:P507-13. doi: 10.1016/S0140-67362030211-7

3. Zhang X, Tan Y, Ling Y, Lu G, Liu F, Yi Z, et al. Viral and host factors related to the clinical outcome of COVID-19. Nature. (2020). doi: 10.1038/s41586-020-2355-0. [Epub ahead of print].

4. Sun Y, Dong Y, Wang L, Xie H, Li B, Chang C, et al. Characteristics and prognostic factors of disease severity in patients with COVID-19: The Beijing experience. J Autoimmun. (2020) 24:102473. doi: 10.1016/j.jaut.2020.102473

5. Hong KH, Choi JP, Hong SH, Lee J, Kwon JS, Kim SM, et al. Predictors of mortality in Middle East respiratory syndrome (MERS). Thorax. (2018) 73:286-9. doi: 10.1136/thoraxjnl-2016-209313

6. Huang KJ, Su IJ, Theron M, Wu YC, Lai SK, Liu CC, et al. An interferongamma-related cytokine storm in SARS patients. J Med Virol. (2005) 75:18594. doi: 10.1002/jmv.20255 release in patients with HLH (20). A prospective randomized study has shown the promising efficacy of ruxolitinib in the treatment of severe COVID-19 (21). In this trial, the ruxolitinib group showed a significant decrease of levels of 7 cytokines compared to the control group, suggesting ruxolitinib suppress the cytokine storm in patients with severe COVID-19. Patients in the ruxolitinib group also had a faster chest CT improvement and a faster recovery from lymphopenia. Ruxolitinib was also well-tolerated in patients with severe COVID-19, indicating ruxolitinib could be safely used to treat patients with COVID-19 (21). Additionally, therapeutic plasma exchange can reduce the plasma cytokine concentrations rapidly, and has been successfully used to treat HLH and CRS related to CAR T cells infusion $(22,23)$, suggesting plasma exchange may be a reasonable option for severe patients with cytokine storm. In a preliminary study, therapeutic plasma exchange reduced the plasma IL-6 level and improved the oxygenation status in patients with severe COVID-19 who had ARDS (24).

\section{CONCLUSION}

Although we admit that supportive care and antiviral therapy remain the mainstay for treating patients with COVID19, we recommend that treatments for controlling cytokine storm including tocilizumab, etoposide, ruxolitinib, and plasma exchange should be considered in selected COVID-19 patients with cytokine storm. Some pilot studies have shown promising results. Some other treatments may also be effective in controlling the cytokine storm. More randomized clinical trials are needed to evaluate if these treatments could reduce the mortality of patients with COVID-19.

\section{AUTHOR CONTRIBUTIONS}

YM, LF, and J-YL drafted the manuscript. All authors contributed to the article and approved the submitted version.

7. Channappanavar R, Perlman S. Pathogenic human coronavirus infections: causes and consequences of cytokine storm and immunopathology. Semin Immunopathol. (2017) 39:529-39. doi: 10.1007/s00281-017-0629-x Epub 2017 May 2

8. Xu XJ, Tang YM. Cytokine release syndrome in cancer immunotherapy with chimeric antigen receptor engineered T cells. Cancer Lett. (2014) 343:1728. doi: 10.1016/j.canlet.2013.10.004

9. Le RQ, Li L, Yuan W, Shord SS, Nie L, Habtemariam BA, et al. FDA approval summary: tocilizumab for treatment of chimeric antigen receptor $\mathrm{T}$ cellinduced severe or life-threatening cytokine release syndrome. Oncologist. (2018) 23:943-7. doi: 10.1634/theoncologist.2018-0028

10. Diao B, Wang C, Tan Y, Chen X, Liu Y, Ning L, et al. Reduction and functional exhaustion of T cells in patients with coronavirus disease 2019 (COVID-19). Front Immunol. (2020) 11:827. doi: 10.3389/fimmu.2020.00827

11. Mazzoni A, Salvati L, Maggi L, Capone M, Vanni A, Spinicci M, et al. Impaired immune cell cytotoxicity in severe COVID-19 is IL-6 dependent. J Clin Invest. (2020) 28:138554. doi: 10.1172/JCI138554

12. Morena V, Milazzo L, Oreni L, Bestetti G, Fossali T, Bassoli C, et al. Offlabel use of tocilizumab for the treatment of SARS-CoV-2 pneumonia in Milan, Italy. Eur J Intern Med. (2020) 76:36-42. doi: 10.1016/j.ejim.2020. 05.011 
13. Campins L, Boixeda R, Perez-Cordon L, Aranega R, Lopera C, Force L. Early tocilizumab treatment could improve survival among COVID-19 patients. Clin Exp Rheumatol. (2020) 38:578.

14. Levi M. Tocilizumab for severe COVID-19: a promising intervention affecting inflammation and coagulation. Eur J Intern Med. (2020) 76:2122. doi: 10.1016/j.ejim.2020.05.018

15. Xu X, Han M, Li T, Sun W, Wang D, Fu B, et al. Effective treatment of severe COVID-19 patients with tocilizumab. Proc Natl Acad Sci USA. (2020) 117:10970-5. doi: 10.1073/pnas.2005615117

16. La Rosee P. Treatment of hemophagocytic lymphohistiocytosis in adults. Hematology Am Soc Hematol Educ Program. (2015) 2015:190-6. doi: 10.1182/asheducation-2015.1.190

17. Channappanavar R, Fehr AR, Vijay R, Mack M, Zhao J, Meyerholz DK, et al. Dysregulated type I interferon and inflammatory monocyte-macrophage responses cause lethal pneumonia in SARS-CoV-infected mice. Cell Host Microbe. (2016) 19:181-93. doi: 10.1016/j.chom.2016.01.007

18. Merad M, Martin JC. Pathological inflammation in patients with COVID19: a key role for monocytes and macrophages. Nat Rev Immunol. (2020) 20:355-62. doi: 10.1038/s41577-020-0331-4

19. Zhou YG, Fu BQ, Zheng XH, Wang DS, Zhao CC, Qi YJ, et al. Pathogenic T-cells and inflammatory monocytes incite inflammatory storms in severe COVID-19 patients. Natl Sci Rev. (2020) 13:nwaa041. doi: 10.1093/nsr/nwaa041

20. Ahmed A, Merrill SA, Alsawah F, Bockenstedt P, Campagnaro E, Devata $\mathrm{S}$, et al. Ruxolitinib in adult patients with secondary haemophagocytic lymphohistiocytosis: an open-label, single-centre, pilot trial. Lancet Haematol. (2019) 6:e630-e7. doi: 10.1016/S2352-30261930156-5
21. Cao Y, Wei J, Zou L, Jiang T, Wang G, Chen L, et al. Ruxolitinib in treatment of severe coronavirus disease 2019 (COVID-19): A multicenter, single-blind, randomized controlled trial. I Allergy Clin Immunol. (2020). doi: 10.1016/j.jaci.2020.05.019. [Epub ahead of print].

22. Pandey PK, Kaul E, Agarwal N, Goel S. Effectiveness of therapeutic plasma exchange in a critically ill child with secondary hemophagocytic lymphohistiocytosis. Asian J Transfus Sci. (2019) 13:145-7.doi: 10.4103/ajts.AJTS_45_18

23. Xiao X, He X, Li Q, Zhang $H$, Meng J, Jiang $Y$, et al. Plasma exchange can be an alternative therapeutic modality for severe cytokine release syndrome after chimeric antigen receptor-T Cell Infusion: A case report. Clin Cancer Res. (2019) 25:29-34. doi: 10.1158/1078-0432.CCR-1 8-1379

24. Zhang L, Zhai H, Ma S, Chen J, Gao Y. Efficacy of therapeutic plasma exchange in severe COVID-19 patients. Br J Haematol. (2020). 117:94906. doi: $10.1073 /$ pnas. 2004168117

Conflict of Interest: The authors declare that the research was conducted in the absence of any commercial or financial relationships that could be construed as a potential conflict of interest.

Copyright (c) 2020 Miao, Fan and Li. This is an open-access article distributed under the terms of the Creative Commons Attribution License (CC BY). The use, distribution or reproduction in other forums is permitted, provided the original author(s) and the copyright owner(s) are credited and that the original publication in this journal is cited, in accordance with accepted academic practice. No use, distribution or reproduction is permitted which does not comply with these terms. 